\title{
Epigenetic silencing of protocadherin 10 in colorectal cancer (Review)
}

\author{
XIAN ZHONG $^{1,2}$, HONG SHEN $^{1}$, JIANSHAN MAO $^{3}$, JIAWEI ZHANG $^{4}$ and WEIDONG HAN ${ }^{5}$ \\ ${ }^{1}$ Department of Medical Oncology, Second Affiliated Hospital of Zhejiang University School of Medicine, Hangzhou, \\ Zhejiang 310009; ${ }^{2}$ Department of Medical Oncology, Hangzhou Binjiang Hospital, Hangzhou, Zhejiang 310052; \\ ${ }^{3}$ Department of Gastroenterology and ${ }^{4}$ Cancer Institute, Second Affiliated Hospital of Zhejiang University School of Medicine, \\ Hangzhou, Zhejiang 310009; ${ }^{5}$ Department of Medical Oncology, Sir Run Run Shaw Hospital, \\ Zhejiang University School of Medicine, Hangzhou, Zhejiang 310016, P.R. China
}

Received February 3, 2016; Accepted December 20, 2016

DOI: $10.3892 / 01.2017 .5733$

\begin{abstract}
Colorectal cancer (CRC) is one of the most common types of malignant tumor in the world and occurs through a multi-step process resulting from the accumulation of genetic and epigenetic alterations of the genome. Although the molecular mechanisms of the pathogenesis of CRC remain unclear, the inactivation of tumor suppressor genes (TSGs) through promoter methylation serves an important role. Aberrant methylation is a well-defined marker of CRC. At present, the epigenetic silencing of protocadherin 10 (PCDH10) has been identified as an important TSG with key roles in colorectal carcinogenesis, invasion and metastasis as a frequent and early event. Advances in gene methylation detection in tumor tissues and body fluids have led to the development of non-invasive screening methods for CRC. The present study aimed to review the epigenetic alteration of PCDH10 in CRC development, and the potential of PCDH10 to be a non-invasive biomarker for CRC.
\end{abstract}

\section{Contents}

1. Introduction

2. Characteristics and biological functions of PCDH10

3. The role of PCDH10 as a functional TSG in CRC

4. Aberrant promoter methylation and inactivation of PCDH10 in CRC

5. Non-invasive biomarker for CRC diagnosis

6. Conclusion

Correspondence to: Dr Weidong Han, Department of Medical Oncology, Sir Run Run Shaw Hospital, Zhejiang University School of Medicine, 3 Qingchun Road East, Xihu, Hangzhou, Zhejiang 310016, P.R. China

E-mail: hanwd@zju.edu.cn

Key words: DNA methylation, protocadherin 10, tumor suppressor gene, colorectal cancer, tumor biomarker

\section{Introduction}

Colorectal cancer (CRC) is one of the most common types of malignant tumor in the world (1). The development of CRC is a multi-step process resulting from the accumulation of genetic and epigenetic alterations of the genome (2). The loss of genomic stability and subsequent genetic alterations in tumor suppressor genes (TSG) and oncogenes may initiate carcinogenesis and tumor progression (3). A number of genetic aberrations have been identified in CRC, occurring in the $A P C$, V-Ki-ras2 Kirsten rate sarcoma viral oncogene (KRAS) and TP53 genes $(4,5)$. In addition to genetic changes, epigenetic deregulation including aberrant DNA methylation as a marker of cancer has been extensively studied in CRC (6). Epigenetic gene silencing is associated with the onset and progression of various types of cancer, and it is accepted that epigenetic alterations precede genetic changes during tumorigenesis (7). Aberrant methylation of cytosine-guanine $(\mathrm{CpG})$ islands in TSG promoter regions has been previously proposed as a novel candidate cancer biomarker (8).

Protocadherins (PCDHs) are cadherin-associated receptors that serve an important role in the establishment and function of specific cell-cell connections and in tumor development $(9,10)$. Previously, frequent epigenetic silencing of protocadherin 10 (PCDH10) was revealed in colorectal carcinogenesis. PCDH10 has been proposed to be a tumor suppressor gene involved in the processes of growth control, cell invasion and metastasis (11). The present study focuses on previous findings and aims to review the epigenetic alteration of PCDH10 and the possibility of PCDH10 methylation being a biomarker for CRC diagnosis.

\section{Characteristics and biological functions of PCDH10}

PCDHs constitute a major subfamily of the cadherin superfamily (12). The PCDH family may be divided largely into 2 groups, based on their genomic structure: Clustered PCDHs (PCDH $\alpha, \delta$ and $\gamma$ families) constituting gene clusters on a single chromosome and non-clustered PCDHs scattered over different chromosomes $(13,14)$. The majority of non-clustered 
PCDHs are cell-adhesion molecules with 6 or 7 cadherin motifs in their extracellular domain and several cytoplasmic domains. Non-clustered PCDHs may be classified into 3 groups: $\delta 1, \delta 2$ and $\varepsilon$ subgroups (15). All PCDH $\delta$ members contain highly conserved motifs (CM), CM1, 27 amino acids and CM2, 17 amino acids, in their cytoplasmic domains.

The human PCDH10 gene, termed OL-PCDH or KIAA1400, is located at $4 \mathrm{q} 28.3$ on the long arm of chromosome 4 and is a member of the subgroup of PCDH 82 without phosphatase-1 $\alpha$ (PP1 $\alpha$ ) binding domain (RRVTF, CM3) (16). Unlike other PCDH family members expressed predominantly in the nervous system, PCDH10 has been identified to exhibit widespread expression in almost all normal tissue $(17,18)$ and contains 6 extracellular repeats, a transmembrane domain and a unique cytoplasmic domain (19), including CM2, homologous to a laminin-type epidermal growth factor (EGF)-like (LE) domain (17), as demonstrated in Fig. 1A. CM2 is also similar to the C2HC-type zinc-finger or zinc knuckle finger motif (20), and represents a functional interaction domain of PCDH10, which may mediate intracellular signal transduction. Previous studies have demonstrated that PCDH10 is involved in several important biological pathways in different types of tumor cells. Although PCDH10 appears to lack the $\beta$-catenin binding cytoplasmic site present in classical cadherins (21), it may affect the $\mathrm{Wnt} / \beta$-catenin signaling pathway $(22,23)$. It was revealed that PCDH10 induced apoptosis by inhibiting the nuclear factor (NF)- $\mathrm{kB}$ pathway in myeloma cells (24), or by interacting with human brain expressed X-linked 1 in imatinib-induced K562 cell apoptosis (25), indicating the proapoptotic and drug-resistance reversal role of PCDH10. PCDH10 exhibits cell-to-cell adhesion activity with a weak binding ability, suggesting that the cytoplasmic domain may not efficiently stabilize those interactions to facilitate adhesion or may negatively regulate their extracellular adhesions (15). The underlying mechanism is hypothesized to be distinct from that of classical cadherins. PCDH10 interacts with Nck-associated protein 1 (Nap1)/WAVE1, and the PCDH10/Nap1/WAVE1 complex affects actin assembly and subsequently regulates cell migration (26). However, how PCDH10/Nap1/WAVE1 complex controls actin assembly remains unknown. The molecules interacted with or regulated by PCDH10 are described in Table I.

The minimal promoter region of PCDH10 is identified as the segment between nucleotides -144 and -99 , containing a CAAT box and a GT box, which are required for promoter activity, and a putative transcription factor binding site for AP-4. Specificity protein (Sp) $1 / \mathrm{Sp} 3$ and core binding factor (CBF)/NF-Y transcription factors are crucial to the basal expression of PCDH10 (27). Previously, PCDH10 was revealed to be a transcriptional target of tumor protein (p) 53 and that the levels of PCDH10 expression may be induced by wild type p53 but not mutant p53 in a number of human cancer cell lines (28), suggesting that PCDH10 may be involved in the signaling pathway of p53 regulation, as illustrated in Fig. 1B. Yu et al (29) identified cyclin dependent kinase inhibitor 1A (CDKN1A), FGFR2 and HIV-1 Tat interactive protein 2 (HTATIP2) as the upregulated target genes in a PCDH10 re-expressing gastric cancer cell line by complementary DNA microarray analysis, and hypothesized
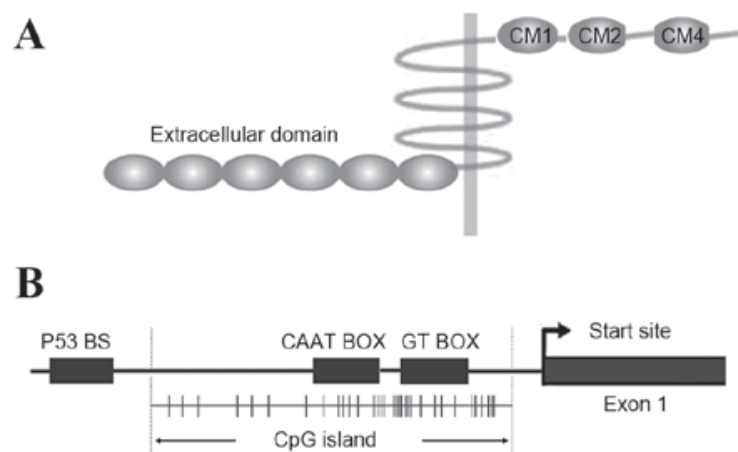

Figure 1. Characteristics of PCDH10. (A) Protein structure of PCDH10. PCDH10 possesses 6 extracellular domains, a transmembrane domain and 3 cytoplasmic domains: CM1, CM2 and CM4. (B) $\mathrm{CpG}$ island and promoter region of $\mathrm{PCDH} 10 . \mathrm{CpG}$ sites are shown as short vertical lines. The transcription start site is indicated by a curved arrow. CAAT and GT BOX exist in the core promoter. A p53 binding site P53 BS is also located in the PCDH10 promoter region, which is responsive to $\mathrm{p} 53$ regulation. $\mathrm{PCDH} 10$, protocadherin 10; CM, cytoplasmic motif; p53, tumor protein 53; BS, binding site; $\mathrm{CpG}$, cytosine-guanine site.

the molecular events mediated by PCDH10, including induction of apoptosis, control of cell growth and inhibition of invasion and metastasis. The signaling pathways involved in PCDH10 regulation are illustrated in Fig. 2. The comprehensive functions of PCDH10 require additional investigation.

\section{Role of PCDH10 as a functional TSG in CRC}

Previously, an association between PCDH10 and tumor development has been proposed. PCDH10 downregulation is involved into several types of cancer, including gastric, hepatocellular, colorectal, breast, cervical, lung, nasopharyngeal, esophageal, pancreatic and bladder cancer (30-35). Functional studies have also identified PCDH10 as a potential novel tumor suppressor gene in multiple types of cancer $(18,32,33)$. The present study demonstrated that PCDH10 may suppress cell proliferation and invasion in colorectal cancer RKO cells. Flow cytometry analysis of PCDH10-re-expressed RKO cells revealed a significant decrease in the number of cells in the G2/M phase, and an increase in the number of cells in the G0/G1 phase, without inducing apoptosis (11). The ectopic expression of PCDH10 significantly reduced the colony formation efficiencies of the HCT116 cell line in monolayer culture and soft-agar assays in vitro (18) and suppressed tumorigenesis and liver metastasis in vivo, resulting in prolonged mice survival (36). Thus, PCDH10 may be an effective therapeutic target for the treatment of $\mathrm{CRC}$ and the mechanism requires attention.

\section{Aberrant promoter methylation and inactivation of PCDH10 in CRC}

DNA methylation represents one of the most studied types of epigenetic marker in CRC (37). The methylation of $\mathrm{CpG}$ islands in the gene promoter region may induce chromatin conformational modifications and inhibit the access of the transcriptional machinery, thus altering gene expression levels. The downregulation of cadherins or their functional alterations 
Table I. Molecule/complex that interact with or are regulated by PCDH10.

\begin{tabular}{llr}
\hline Molecule/complex & \multicolumn{1}{c}{ Functions } & Ref \\
\hline Nap1,WAVE1 & Actin assembly/cell migration & 22 \\
Wnt/ $\beta$-catenin pathway & Cell cycle/apoptosis, carcinogenesis & 18,19 \\
CDKN1A, FGFR2, and HTATIP2 & Apoptosis, growth, invasion and metastasis & 24 \\
NF-kappaB pathway & Apoptosis & 20 \\
hBex1 & Drug resistance & 21 \\
\hline
\end{tabular}

NAP1/Wave1, Nck-associated protein 1/WAVE1; CDKN1A, cyclin dependent kinase inhibitor 1A; HTATIP2, HIV-1 Tat interactive protein 2; $\mathrm{NF}-\kappa \mathrm{B}$, nuclear factor-kappaB.

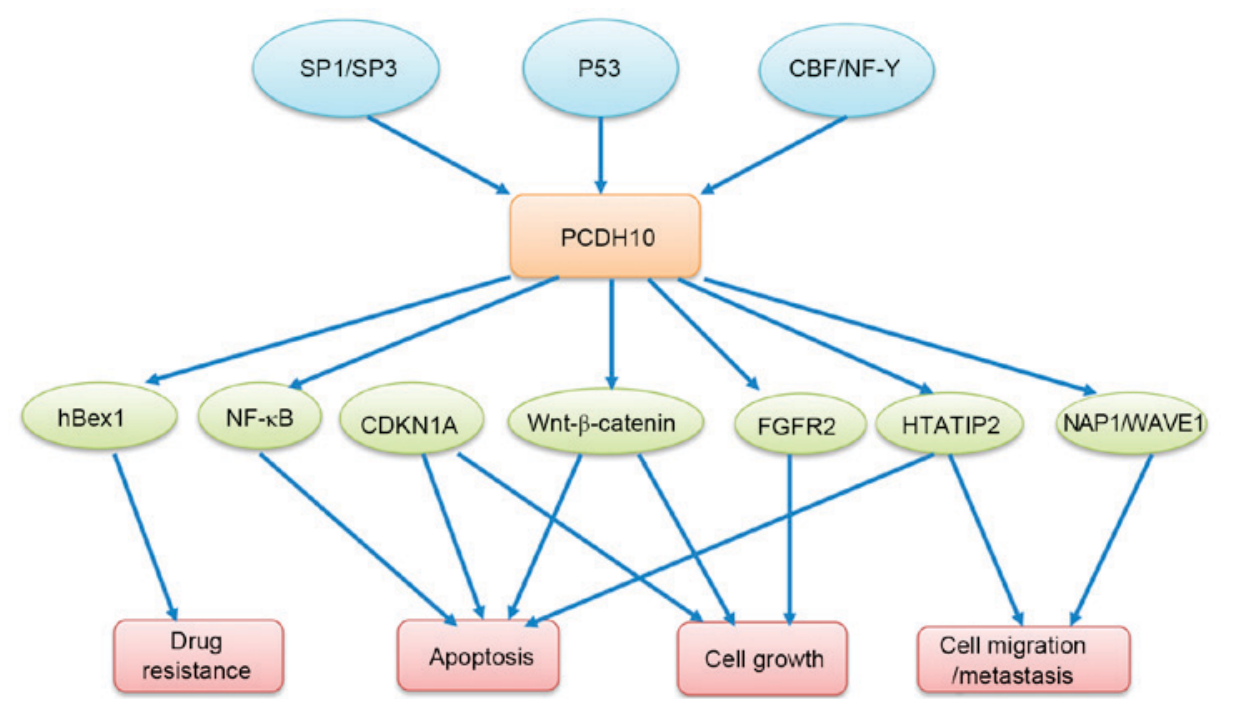

Figure 2. Molecular events for induction of cell apoptosis, reversal of drug resistance, suppression of cell growth and migration/invasion mediated by PCDH10. $\mathrm{Sp1} / \mathrm{Sp} 3$, specificty protein 1/3; p53, tumor protein 53; CBF/NF-Y, core binding factor/nuclear transcription factor Y; NF-kB, nuclear factor-kappa B; CDKN1A, cyclin dependent kinase inhibitor 1A; HTATIP2, HIV-1 Tat interactive protein 2; NAP1/Wave1, Nck-associated protein 1/WAVE1.

have been frequently observed in human malignancies (38). Several studies have demonstrated the aberrant methylation of the PCDH10 promoter region in a wide spectrum of types of cancer by various techniques, such as methylation-specific polymerase chain reaction (MSP PCR) and MSP quantitative (q) PCR and high-resolution melting (HRM) analysis (35). Promoter methylation and transcriptional silencing of PCDH10 were detected in all analyzed colorectal carcinoma cell lines, but not in immortalized normal epithelial cell lines (11). Ying et al (18) demonstrated that the transcriptional silencing of PCDH10 may be reversed by pharmacologic demethylation with 5-aza-2'-deoxycytidine or genetic demethylation with double knockout of DNA methyltransferase (DNMT) 1 and DNMT3B in the colorectal cancer HCT116 cell line, suggesting a direct epigenetic mechanism (18). The aberrant methylation of the PCDH10 promoter was also observed in $43-85 \%$ of colorectal cancer tissues in several studies, indicating that PCDH10 methylation is a frequent event in colorectal carcinogenesis $(11,35,39)$. In the present study, PCDH10 methylation was detected in colorectal cancer samples, but not in paired adjacent colorectal tissues, in accordance with the study by Yu et al (35), suggesting that the aberrant promoter methylation of PCDH10 is specific to colorectal cancer cells.
As recognized previously, candidate TSGs may be inactivated through epigenetic inactivation, biallelic genetic inactivation or the two (7). Notably, the allelic loss of PCDH10 was revealed in 53/171 types of colorectal carcinoma and the expression of the PCDH10 gene was silenced or markedly downregulated in 41 of the aforementioned 53 types of colorectal carcinoma compared with their matched normal mucosa, indicating genetic deletion may be an additional mechanism for PCDH10 inactivation in CRC (36), although Ying et al (18) did not detect any homozygous deletion of the PCDH10 gene in CRC cell lines. This suggests that the epigenetic inactivation and genetic alteration of PCDH10 serve important roles in the development of CRC. Additional study is required to assess whether other types of genetic inactivation, including point mutations, are involved in colorectal tumorigenesis.

\section{Non-invasive biomarker for CRC diagnosis}

CRC prognosis remains poor, and the 5-year survival rates are $\sim 90 \%$ for patients with early stage disease but decrease to $<10 \%$ in patients with distant metastases (40). The early detection of CRC through screening programs that detect mucosal 
changes that are predictive of colorectal tumors may reduce the incidence and mortality rates of this disease $(41,42)$. However, novel biomarkers that are absent in the healthy population and present in patients with CRC need to be identified, particularly those that may be detected at the early development stage of the disease and applied in screening tests. Since methylated genes that are present in tumor tissues may be identified in serum/plasma, stool or ascitic fluid, epigenetic biomarkers represent a well-suited biological material for a non-invasive screening method for CRC diagnosis (43). Using the MSP qPCR method, Danese et al (44) demonstrated that the PCDH10 promoter methylation was detected in $94.0 \%$ of surgically resected tumors and in $62.7 \%$ of plasma samples, with a high concordance rate between tissues and plasma samples of $66.6 \%$ in total, and $76.3 \%$ in patients with early (stage I/II) CRC (45), demonstrating a significant correlation between PCDH10 methylation in cell-free DNA and tumor tissue. Since methylated alleles may be detected with a high degree of sensitivity, there is great scope in using methylation as a potential early detection system for CRC. Stool DNA is another well-suited biological material for $\mathrm{CRC}$ diagnosis. The methylated PCDH10 promoter sequence in stool as a biomarker may be investigated in additional studies. With respect to CRC diagnosis, fecal occult blood tests (FOBT) are a commonly used non-invasive screening procedure, which reduce $\mathrm{CRC}$-associated mortality by $20 \%$ when performed every 2 years (46). Detecting PCDH10 methylation in plasma and/or stool, combined with FOBT, may be a good diagnostic regimen to improve the sensitivity and effectiveness. Additional case-control studies are required to address this issue.

Whether PCDH10 may be a prognostic biomarker has also been investigated. However, a significant association has not been revealed between PCDH10 methylation status and tumor node metastasis staging, DFS or OS in CRC (35). A possible reason for this is that $\mathrm{PCDH} 10$ promoter methylation is an early event in CRC carcinogenesis, and is hypothesized to be the cause, rather than the outcome, of carcinogenesis. Additionally, the different extent, full or partial, of promoter methylation may affect the gene expression level and function. Thus, it is important to determine the presence and extent of gene methylation. Jao et al (36) reported that genetic and/or epigenetic alterations contribute to the PCDH10 inactivation that occurs in the majority of CRC tumors, and the genetic aberration of PCDH10 was demonstrated to be significantly associated with tumor progression and distant metastasis, indicating it may be an independent predictor of poor survival rates for patients with CRC. Heitzer et al (47) found that the promoter methylation statuses of PCDH10, SPARC and UCHL1 may be used as prognostic and predictive molecular markers for patients with CRC, and facilitate treatment decisions for stage II colorectal cancer. These results suggest that genetic changes or epigenetic aberrations of PCDH10, combined with other biomarkers, may exhibit prognostic value for CRC.

\section{Conclusion}

Aberrant methylation of the PCDH10 promoter, causing gene inactivation, is a frequent and early event in CRC. PCDH10 has been identified as an important TSG, with key roles in colorectal carcinogenesis, invasion and metastasis. PCDH10 methylation status may be a valuable non-invasive biomarker for the diagnosis of CRC.

\section{Acknowledgements}

The present study was supported by Health Program of Hangzhou (grant no. 20140633B26), National Natural Science Foundation of China (grant nos. 81000945, 81071619, 81101477) and the Zhejiang Provincial Natural Science Foundation of China (grant no. Y2110018).

\section{References}

1. Siegel RL, Miller KD and Jemal A: Cancer statistics, 2015. CA Cancer J Clin 65: 5-29, 2015.

2. Grady WM and Carethers JM: Genomic and epigenetic instability in colorectal cancer pathogenesis. Gastroenterology 135: 1079-1099, 2008.

3. Bach SP, Renehan AG and Potten CS: Stem cells: The intestinal stem cell as a paradigm. Carcinogenesis 21: 469-476, 2000.

4. ChoKR and VogelsteinB: Geneticalterationsintheadenoma-carcinoma sequence. Cancer 70 (6 Suppl): S1727-S1731, 1992.

5. Zoratto F, Rossi L, Verrico M, Papa A, Basso E, Zullo A, Tomao L, Romiti A, Lo Russo G and Tomao S: Focus on genetic and epigenetic events of colorectal cancer pathogenesis: Implications for molecular diagnosis. Tumour Biol 35: 6195-6206, 2014.

6. Wong JJ, Hawkins NJ and Ward RL: Colorectal cancer: A model for epigenetic tumorigenesis. Gut 56: 140-148, 2007.

7. Jones PA and Baylin SB: The fundamental role of epigenetic events in cancer. Nat Rev Genet 3: 415-428, 2002.

8. Kim MS, Lee J and Sidransky D: DNA methylation markers in colorectal cancer. Cancer Metastasis Rev 29: 181-206, 2010.

9. Yang X, Chen MW, Terry S, Vacherot F, Chopin DK, Bemis DL, Kitajewski J, Benson MC, Guo Y and Buttyan R: A human- and male-specific protocadherin that acts through the wnt signaling pathway to induce neuroendocrine transdifferentiation of prostate cancer cells. Cancer Res 65: 5263-5271, 2005.

10. Waha A, Güntner S, Huang TH, Yan PS, Arslan B, Pietsch T, Wiestler OD and Waha A: Epigenetic silencing of the protocadherin family member PCDH-gamma-A11 in astrocytomas. Neoplasia 7: 193-199, 2005.

11. Zhong X, Zhu Y, Mao J, Zhang J and Zheng S: Frequent epigenetic silencing of PCDH10 by methylation in human colorectal cancer. J Cancer Res Clin Oncol 139: 485-490, 2013.

12. Sano K, Tanihara H, Heimark RL, Obata S, Davidson M, St John T, Taketani S and Suzuki S: Protocadherins: A large family of cadherin-related molecules in central nervous system. EMBO J 12: 2249-2256, 1993.

13. Morishita $\mathrm{H}$ and Yagi T: Protocadherin family: Diversity, structure, and function. Curr Opin Cell Biol 19: 584-592, 2007.

14. Wu Q and Maniatis T: A striking organization of a large family of human neural cadherin-like cell adhesion genes. Cell 97: 779-790, 1999.

15. Kim SY, Yasuda S, Tanaka H, Yamagata K and Kim H: Non-clustered protocadherin. Cell Adh Migr 5: 97-105, 2011.

16. Vanhalst K, Kools P, Staes K, van Roy F and Redies C: delta-Protocadherins: A gene family expressed differentially in the mouse brain. Cell Mol Life Sci 62: 1247-1259, 2005.

17. Wolverton $\mathrm{T}$ and Lalande $\mathrm{M}$ : Identification and characterization of three members of a novel subclass of protocadherins. Genomics 76: 66-72, 2001.

18. Ying J,Li H, Seng TJ, Langford C, Srivastava G, Tsao SW, Putti T, Murray P, Chan AT and Tao Q: Functional epigenetics identifies a protocadherin $\mathrm{PCDH} 10$ as a candidate tumor suppressor for nasopharyngeal, esophageal and multiple other carcinomas with frequent methylation. Oncogene 25: 1070-1080, 2006.

19. Hirano S, Yan Q and Suzuki ST: Expression of a novel protocadherin, OL-protocadherin, in a subset of functional systems of the developing mouse brain. J Neurosci 19: 995-1005, 1999.

20. Matthews JM, Kowalski K, Liew CK, Sharpe BK, Fox AH, Crossley M and MacKay JP: A class of zinc fingers involved in protein-protein interactions biophysical characterization of CCHC fingers from fog and U-shaped. Eur J Biochem 267: 1030-1038, 2000. 
21. Frank M and Kemler R: Protocadherins. Curr Opin Cell Biol 14 557-562, 2002.

22. Zhao Y, Yang Y, Trovik J, Sun K, Zhou L, Jiang P, Lau TS, Hoivik EA, Salvesen HB, Sun H and Wang H: A novel wnt regulatory axis in endometrioid endometrial cancer. Cancer Res 74: 5103-5117, 2014.

23. Xu Y, Yang Z, Yuan H, Li Z, Li Y, Liu Q and Chen J: PCDH10 inhibits cell proliferation of multiple myeloma via the negative regulation of the Wnt/ $\beta$-catenin/BCL-9 signaling pathway. Oncol Rep 34: 747-754, 2015.

24. Li Z, Yang Z, Peng X, Li Y, Liu Q and Chen J: Nuclear factor- $\kappa \mathrm{B}$ is involved in the protocadherin-10-mediated pro-apoptotic effect in multiple myeloma. Mol Med Rep 10: 832-838, 2014

25. Ding K, Su Y, Pang L, Lu Q, Wang Z, Zhang S, Zheng S, Mao J and Zhu Y: Inhibition of apoptosis by downregulation of hBex1, a novel mechanism, contributes to the chemoresistance of $\mathrm{Bcr} / \mathrm{Abl}+$ leukemic cells. Carcinogenesis 30: 35-42, 2009.

26. Nakao S, Platek A, Hirano S and Takeichi M: Contact-dependent promotion of cell migration by the OL-protocadherin-Nap1 interaction. J Cell Biol 182: 395-410, 2008.

27. Li Z, Xie J, Li W, Tang A, Li X, Jiang Z, Han Y, Ye J, Jing J, Gui Y and Cai Z: Identification and characterization of human PCDH10 gene promoter. Gene 475: 49-56, 2011.

28. Shi D, Murty VV and Gu W: PCDH10, a novel p53 transcriptional target in regulating cell migration. Cell Cycle 14: 857-866, 2015.

29. Yu J, Cheng YY, Tao Q, Cheung KF, Lam CN, Geng H, Tian LW, Wong YP, Tong JH, Ying JM, et al: Methylation of protocadherin 10 , a novel tumor suppressor, is associated with poor prognosis in patients with gastric cancer. Gastroenterology 136: 640-651. e1, 2009.

30. Fang S, Huang SF, Cao J, Wen YA, Zhang LP and Ren GS: Silencing of PCDH10 in hepatocellular carcinoma via de novo DNA methylation independent of HBV infection or HBX expression. Clin Exp Med 13: 127-134, 2013.

31. Lin YL, Li ZG, He ZK, Guan TY and Ma JG: Clinical and prognostic significance of protocadherin-10 (PCDH10) promoter methylation in bladder cancer. J Int Med Res 40: 2117-2123, 2012.

32. Narayan G, Scotto L, Neelakantan V, Kottoor SH, Wong AH, Loke SL, Mansukhani M, Pothuri B, Wright JD, Kaufmann AM, et al: Protocadherin PCDH10, involved in tumor progression, is a frequent and early target of promoter hypermethylation in cervical cancer. Genes Chromosomes Cancer 48 983-992, 2009

33. Tang X, Yin X, Xiang T, Li H, Li F, Chen L and Ren G: Protocadherin 10 is frequently downregulated by promoter methylation and functions as a tumor suppressor gene in non-small cell lung cancer. Cancer Biomark 12: 11-19, 2012.
34. Wang L, Xie PG, Lin YL, Ma JG and Li WP: Aberrant methylation of PCDH10 predicts worse biochemical recurrence-free survival in patients with prostate cancer after radical prostatectomy. Med Sci Monit 20: 1363-1368, 2014.

35. Yu B, Yang H, Zhang C, Wu Q, Shao Y, Zhang J, Guan M, Wan J and Zhang W: High-resolution melting analysis of PCDH10 methylation levels in gastric, colorectal and pancreatic cancers. Neoplasma 57: 247-252, 2010.

36. Jao TM, Tsai MH, Lio HY, Weng WT, Chen CC, Tzeng ST, Chang CY, Lai YC, Yen SJ, Yu SL and Yang YC: Protocadherin 10 suppresses tumorigenesis and metastasis in colorectal cancer and its genetic loss predicts adverse prognosis. Int J Cancer 135: 2593-2603, 2014

37. Lao VV and Grady WM: Epigenetics and colorectal cancer. Nat Rev Gastroenterol Hepatol 8: 686-700, 2011.

38. Pignatelli M: Integrins, cadherins, and catenins: Molecular cross-talk in cancer cells. J Pathol 186: 1-2, 1998.

39. Silva TD, Vidigal VM, Felipe AV, DE Lima JM, Neto RA, Saad SS and Forones NM: DNA methylation as an epigenetic biomarker in colorectal cancer. Oncol Lett 6: 1687-1692, 2013.

40. Toribara NW and Sleisenger MH: Screening for colorectal cancer. N Engl J Med 332: 861-867, 1995.

41. Pignone MP and Lewis CL: Using quality improvement techniques to increase colon cancer screening. Am J Med 122: 419-420, 2009.

42. Winawer SJ: The multidisciplinary management of gastrointestinal cancer. Colorectal cancer screening. Best Pract Res Clin Gastroenterol 21: 1031-1048, 2007.

43. Shivapurkar N and Gazdar AF: DNA methylation based biomarkers in non-invasive cancer screening. Curr Mol Med 10: $123-132,2010$.

44. Danese E, Minicozzi AM, Benati M, Montagnana M, Paviati E, Salvagno GL, Gusella M, Pasini F, Guidi GC and Lippi G: Epigenetic alteration: New insights moving from tissue to plasma- the example of PCDH10 promoter methylation in colorectal cancer. Br J Cancer 109: 807-813, 2013.

45. Edge SB, Byrd DR, Compton CC, Fritz AG, Greene FL and Trotti A (eds): AJCC cancer staging manual (7th edition). New York, NY: Springer, 2010.

46. Atkin W: Options for screening for colorectal cancer. Scandin J Gastroenterol 38: 13-16, 2009.

47. Heitzer E, Artl M, Filipits M, Resel M, Graf R, Weißenbacher B, Lax S, Gnant M, Wrba F, Greil R, et al: Differential survival trends of stage II colorectal cancer patients relate to promoter methylation status of PCDH10, SPARC, and UCHL1. Mod Pathol 27: 906-915, 2014. 